\title{
Strongly Balanced 4-Kite Designs Nested into OQ-Systems
}

\author{
Mario Gionfriddo ${ }^{1}$, Lorenzo Milazzo ${ }^{1}$, Rosaria Rota ${ }^{2}$ \\ ${ }^{1}$ Dipartimento di Matematica e Informatica, Universitá di Catania, Catania, Italy \\ ${ }^{2}$ Dipartimento di Matematica, Universitá di Roma Tre, Roma, Italy \\ Email: gionfriddo@dmi.unict.it, milazzo@dmi.unict.it,rota@mat.uniroma3.it
}

Received November 20, 2012; revised December 10, 2012; accepted December 18, 2012

Copyright (C) 2013 Mario Gionfriddo et al. This is an open access article distributed under the Creative Commons Attribution License, which permits unrestricted use, distribution, and reproduction in any medium, provided the original work is properly cited.

\begin{abstract}
In this paper we determine the spectrum for octagon quadrangle systems [OQS] which can be partitioned into two strongly balanced 4-kitedesigns.
\end{abstract}

Keywords: Graphs; Designs; 4-Kite

\section{Introduction}

Let $K=(X, \mathcal{E})$ be a graph defined on the vertex set $X$. Let $G$ be a subgraph of $K$. A $G$-decomposition of $K$ is a pair $\Sigma=(X, \mathcal{B})$, where $\mathcal{B}$ is a partition of the edge set of $K$ into subsets isomorphic to $G$. If $K_{v}$ is the complete undirected graph defined on the vertex set $X$, a $G$-decomposition $\Sigma=(X, \mathcal{B})$ of $K_{v}$ is called a $G$-design of order $v$ and the classes of the partition $\mathcal{B}$ are said to be the blocks of $\Sigma$. A $G$-design is called balanced if for each vertex $x \in X$, the number of blocks of $\Sigma$ containing $x$ is a constant. Observe that if $G$ is a regular graph then a $G$-design is always balanced, hence the notion of a balanced $G$-design becomes meaningful only for a non-regular graph $G$.

Let $G$ be a graph and let $A_{1}, A_{2}, \cdots, A_{h}$ be the orbits of the automorphism group of $G$ on its vertex-set. Let $\Sigma=(X, \mathcal{B})$ be a $G$-design. We define the degree $d_{A_{i}}(x)$ of a vertex $x \in X$ as the number of blocks of $\Sigma$ containing $x$ as an element of $A_{i}$. We say that $\Sigma=(X, \mathcal{B})$ is a strongly balanced $G$-design if, for every $i=1,2, \cdots, h$, there exists a constant $C_{i}$ such that $d_{A_{i}}(x)=C_{i}$, for every $x \in X$ [1-3]. Clearly, since for each vertex $x \in V$ the relation $d(x)=\sum_{i=1}^{h} d_{A_{i}}(x)$ holds, we have that "a strongly balanced G-design is always a balanced G-design". We say that a $G$-design is simply balanced if it is balanced, but not strongly balanced.

A cycle of length 4 with a pendant edge, i.e. the graph $C_{4}+e$, is called a 4-kite and is denoted by $(a, b, c, d)-e$ or $(c, b, a, d)-e$, where $\{a, b\},\{b, c\},\{c, d\},\{d, a\}$, $\{d, e\}$ are the edges of the 4-kite. Note that a cycle of length 3 with a pendant edge is called a 3-kite or just a kite. In the case when $G$ is a 4-kite, a $G$-design is called a 4-kite-design or also a 4-kite-system. It is known that a 4-kite design of order $v$ exists when $v \equiv 0$ or $1(\bmod 5)$. Further research on 4-kite designs can be found in [2]. We will call the vertices $a$ and $c$ of the 4-kite $(a, b, c, d)-e$ the lateral vertices, $b$ the middle vertex, $d$ the center vertex and $e$ the terminal vertex [4-6]. Some balanced $G$ designs, when $G$ is a path, have been studied in $[1,3]$. Strongly balanced $G$-designs were first introduced in [1], in which the spectrum of simply balanced and strongly balanced $P_{5}$ and $P_{6}$-designs have been determined, where $P_{k}$ denotes a path with $k$ vertices.

An octagon quadrangle is a graph denoted by $\left[\left(x_{1}\right), x_{2}, x_{3},\left(x_{4}\right),\left(x_{5}\right), x_{6}, x_{7},\left(x_{8}\right)\right]$ and formed by an 8cycle $\left(x_{1}, x_{1}, x_{2}, \cdots, x_{8}\right)$ with the two additional edges $\left\{x_{1}, x_{4}\right\}$ and $\left\{x_{5}, x_{8}\right\}$. An octagon quadrangle system [OQS] is a $G$-design, where $G$ is an octagon quadrangle. OQS $(v)$ s have been defined and studied in [4,7-9]. In these papers, the main idea was to follow the research about hexagon triangle systems and all the others already introduced in the literature, where we can find many authors who have studied in many ways polygon triangle systems using triangulations of polygons [5,10,11]. With the study of octagon quadrangles the authors have considered quadrangulations of polygons with new ideas for the research $[12,13]$.

In what follows, if $\Sigma=(X, \mathcal{B})$ is an $\operatorname{OQS}(v)$ in which the family of all $K_{1}(Q)$ contained in the blocks of $\mathcal{B}$ forms a 4-kite design $\mathcal{K}=(X, \mathcal{C})$, we will say that $\Sigma$ is nesting $\mathcal{K}$ or also that $\mathcal{K}$ is nested in $\Sigma$. Similar problems, including colorings, can be found also in $[14,15]$.

In this paper, starting from the remark that an octagon 
quadrangle $Q=\left[\left(x_{1}\right), x_{2}, x_{3},\left(x_{4}\right),\left(x_{5}\right), x_{6}, x_{7},\left(x_{8}\right)\right]$, can be partitioned into two 4-kites

$$
\begin{aligned}
& K_{1}(Q)=\left(x_{1}, x_{2}, x_{3}, x_{4}\right)-\left(x_{5}\right), \\
& K_{2}(Q)=\left(x_{5}, x_{6}, x_{7}, x_{8}\right)-\left(x_{1}\right),
\end{aligned}
$$

the authors study OQSs which can be partitioned into two strongly balanced $\left(C_{4}+e\right)$-designs, determining their spectrum.

\section{Necessary Existence Conditions}

If $\Sigma=(X, \mathcal{B})$ is strongly balanced 4-kite design, its vertices describe four orbits in the automorphism group of a block, which is a graph $C_{4}+e$. We will indicate by $C$ the number of blocks containing any vertex as a center of the 4-kite block, by $T$ the number of blocks containing any vertex as a terminal, by $L$ and $M$ the number of blocks containing any vertex as lateral or median, respectively.

In this section, we determine necessary conditions for the existence of strongly balanced 4-kite designs (order $v$, index $\mu$ ) and for the existence of OQS (order $v$, index $\lambda$ ) nesting strongly balanced 4-kite designs. These conditions are preliminary for conclusive Theorems of Section 3.

Theorem 2.1. If $\Sigma=(X, \mathcal{B})$ is a strongly balanced 4-kite design of order $v$ and index $\mu$, then:

1) $C=T=M=\mu \cdot \frac{v-1}{10}$;

2) $L=\mu \cdot \frac{v-1}{5}$;

3) $\mu \cdot(v-1) \equiv 0, \bmod 10$.

Proof. If $\Sigma=(X, \mathcal{B})$ is a strongly balanced 4-kite design of order $v$ and index $\mu$, following the terminology described above and considering that each vertex occupies $C$ times the central position in the blocks, necessarily:

$$
|\mathcal{B}|=v \cdot C,
$$

from which $C=\mu \cdot \frac{v-1}{10}$.
The same considerations can be done to calculate the parameters $T, M$, which have the same value of $C$. For the last parameter $L$, we can consider that:

$$
3 C+T+2 M+2 L=\mu \cdot(v-1),
$$

hence

$$
L=\mu \cdot \frac{v-1}{5} .
$$

Thus, 1) and 2) are verified and from them 3) holds.

Theorem 2.2. Let $\Sigma=(X, \mathcal{B})$ be an OQS of order $v$ and index $\lambda$, nesting a strongly balanced 4-kite design $\mathcal{K}=(X, \mathcal{C})$ of index $\mu$. Then:

1) $\lambda=2 \cdot \mu$

2) if $\mu=1$ and $\lambda=2$, then $v \equiv 1, \bmod 10$.

Proof. 1) Since:

$$
|\mathcal{B}|=\frac{\lambda \cdot v \cdot(v-1)}{20},|\mathcal{C}|=\frac{\mu \cdot v \cdot(v-1)}{10}
$$

and necessarily $|\mathcal{B}|=|\mathcal{C}|$, it follows $\lambda=2 \cdot \mu$. 2) From 3) of Theorem 2.1 , if $\mu=1$ then $v \equiv 1, \bmod 10$.

\section{Main Existence Theorems}

In what follows, if $B=[(a), b, c,(d),(\alpha), \beta, \gamma,(\delta)]$ is a block of an $O Q$-system $\Sigma$ defined in $Z_{v}$, then the translates of $B$ are all the blocks of type

$$
\begin{aligned}
B_{j}= & {[(a+j), b+j, c+j,(d+j),(\alpha+j),} \\
& \beta+j, \gamma+j,(\delta+j)],
\end{aligned}
$$

for every $j \in Z_{v}$. B is called a base block of $\Sigma$.

Theorem 3.1. There exists an OQS, of order $v$ and index two, nesting a strongly balanced 4-kite design of index one if and only if:

$$
v \equiv 1, \bmod 10, v \geq 11 \text {. }
$$

Proof. $\Rightarrow$ Let $\Sigma=\left(Z_{v}, \mathcal{B}\right)$ be an OQS of order $v$ and index $\lambda$, nesting a 4-kite design $\mathcal{K}=\left(Z_{v}, \mathcal{C}\right)$ of order $v$ and index one. From Theorem 2.2 it is $\lambda=2$ and

$$
v \equiv 1, \bmod 10, v \geq 11 \text {. }
$$

$\Leftarrow$ Consider the following octagon quadrangles:

$$
\begin{gathered}
B_{1}=[(0), h, 4 h+1,(h+1),(6 h+1), 8 h+1,7 h+1,(2 h+1)], \\
B_{2}=[(0), h-1,4 h+1,(h+2),(6 h+1), 8 h, 7 h+1,(2 h+2)], \\
B_{3}=[(0), h-2,4 h+1,(h+3),(6 h+1), 8 h-1,7 h+1,(2 h+3)], \\
\vdots \\
B_{i}=[(0), h-i-1,4 h+1,(h+i),(6 h+1), 8 h-(i-2), 7 h+1,(2 h+i)], \\
\vdots \\
B_{h-1}=[(0), 2,4 h+1,(2 h-1),(6 h+1), 7 h+3,7 h+1,(3 h-1)], \\
B_{h}=[(0), 1,4 h+1,(2 h),(6 h+1), 7 h+2,7 h+1,(3 h)] .
\end{gathered}
$$


Consider the system $\Sigma=(X, \mathcal{B})$, defined in $X=Z_{v}$, having $B_{1}, \cdots, B_{i}, \cdots, B_{h}$ as base blocks. This means that the blocks $B_{1}, B_{2}, \cdots, B_{i}, \cdots, B_{h}$ belong to $\mathcal{B}$ and with all their translates.

Observe that, for $h=1$, the correspondent system defined in $Z_{11}$ has for blocks all the translates of the following base block:

$$
B=[(0), 1,5,(2),(7), 9,8,(3)] .
$$

In every case, it is possible to verify that $\Sigma$ is an $O Q S$ of order $v=10 h+1$ and index $\lambda=2$. Further, if we partition every block

$$
Q=\left[\left(x_{1}\right), x_{2}, x_{3},\left(x_{4}\right),\left(x_{5}\right), x_{6}, x_{7},\left(x_{8}\right)\right],
$$

into the two 4-kites:

$$
\begin{aligned}
& K_{1}(Q)=\left(x_{1}, x_{2}, x_{3}, x_{4}\right)-\left(x_{5}\right), \\
& K_{2}(Q)=\left(x_{5}, x_{6}, x_{7}, x_{8}\right)-\left(x_{1}\right),
\end{aligned}
$$

we can verify that the collection of all the upper 4-kites forms a 4-kite-design $\mathcal{K}_{1}=\left(Z_{v}, \mathcal{C}_{1}\right)$ of index one. Observe also that the collection of all the lower 4-kites forms a 4-kite-design $\mathcal{K}_{2}=\left(Z_{v}, \mathcal{C}_{2}\right)$ of index one.

We can verify that both the systems $\mathcal{K}_{1}, \mathcal{K}_{2}$ are strongly balanced. In fact, for them it is $C=T=M=h$, and $L=2 h$.

To verify this, it is enough to consider that the system $\Sigma$ is constructed by base blocks and difference method.

This proves that $\Sigma$ is an OQS of order $v=10 h+1$, $h \geq 1$, where the two 4-kites designs nested in it have both index one and are both strongly balanced.

At last, it follows a result about the existence of strongly balanced 4-kite designs, whose spectrum is unknown.

Theorem 3.2. For every $v \equiv 1, \bmod 10, v \geq 11$, there exists strongly balanced 4-kite designs of index one.

\section{Conclusive Remarks and Problems}

Theorem 3.1 gives completely the spectrum of $O Q S$ which can be partitioned into two strongly balanced 4kite designs. For a given $v$ belongs to the spectrum, Theorem 3.1 gives also the method to construct an OQS of order $v$ with the said properties.

For example, if $v=21$, the translates of the two base blocks:

$$
\begin{aligned}
& B_{1}=[(0), 2,9,(3),(13), 17,15,(7)], \\
& B_{2}=[(0), 1,9,(4),(13), 16,15,(6)],
\end{aligned}
$$

constructed on $Z_{21}$, define an OQS of order $v=21$ and index $\lambda=2$. We can observe that $B_{1}$ can be partitioned into the two 4-kites

$$
K_{1,1}(Q)=(0,2,9,3)-13, K_{1,2}(Q)=(13,17,15,7)-0,
$$

and $B_{2}$ into the two 4-kites

$$
\begin{aligned}
& K_{2,1}(Q)=(0,1,9,4)-13, \\
& K_{2,2}(Q)=(13,16,15,6)-0 .
\end{aligned}
$$

We can verify that the translates of $K_{1,1}$ and $K_{2,1}$ define a strongly balanced 4-kite designs of order $v=21$ and index $\mu=1$. Further, a system of the same type and parameters is defined by the translates of $K_{1,2}$ and $K_{2,2}$.

The Theorem 3.1 permits also to find values of $v$ for which there exist strongly balanced 4-kite designs, whose spectrum is still unknown. Thus, the statement of Theorem 3.2 can be the starting point for its determination.

In conclusion, we observe that from Theorem 3.1 follows the more general:

Theorem 4.1. There exists an OQS, of order $v$ and index $\lambda$, nesting a strongly balanced 4-kite design of order $v$ and of index $\mu=\lambda / 2$, if and only if:

$$
v \equiv 1, \bmod 10, v \geq 11 \text {. }
$$

Proof. From Theorem 2.2, it is necessarily $\lambda=2 \mu$. So, from Theorem 3.1, by a repetition of blocks, the statement follows.

We can also point out that, after the determination of the spectrum, found in this paper, it is possible to study other problems about octagon quadrangle systems. It is possible to study the intersection problem among them, about which there exist an important literature, following the technique introduced in [16,17]. Also, it should be interesting to examine the conjecture of Berge for linear hypergraphs, in the case in which these are OQSs, following the ideas seen in $[18,19]$.

\section{REFERENCES}

[1] L. Berardi, M. Gionfriddo and R. Rota, "Balanced and Strongly Balanced $\mathrm{P}_{\mathrm{k}}$-Designs," Discrete Mathematics, Vol. 312, No. 3, 2012, pp. 633-636. doi:10.1016/j.disc.2011.05.015

[2] M. Gionfriddo, S. Kucukcifci and L. Milazzo, "Balanced and Strongly Balanced 4-Kite Systems," Utilitas Mathematica, Vol. 90, 2013.

[3] M. Gionfriddo and G. Quattrocchi, "Embedding Balanced $\mathrm{P}_{3}$-Designs into (Balanced) $\mathrm{P}_{4}$-Designs," Discrete Mathematics, Vol. 308, No. 2-3, 2008, pp. 155-160. doi:10.1016/i.disc.2006.11.027

[4] L. Berardi, M. Gionfriddo and R. Rota, "Perfect Octagon Quadrangle Systems with an Upper $\mathrm{C}_{4}$-System and a Large Spectrum," Computer Science Journal of Moldova, Vol. 18, No. 3, 2010, pp. 303-318.

[5] L. Gionfriddo, "Hexagon Kite Systems," Discrete Mathematics, Vol. 309, No. 2, 2009, pp. 505-512. doi:10.1016/j.disc.2008.02.042

[6] M. Gionfriddo and S. Milici, "Octagon Kite Systems," Electronic Notes in Discrete Mathematics, Vol. 41, 2013. 
[7] L. Berardi, M. Gionfriddo and R. Rota, "Perfect Octagon Quadrangle Systems," Discrete Mathematics, Vol. 310, No. 13-14, 2010, pp. 1979-1985. doi:10.1016/j.disc.2010.03.012

[8] L. Berardi, M. Gionfriddo and R. Rota, "Perfect Octagon Quadrangle Systems with Upper $\mathrm{C}_{4}$-Systems," Journal of Statistical Planning and Inference, Vol. 141, No. 7, 2011, pp. 2249-2255. doi:10.1016/j.jspi.2011.01.015

[9] L. Berardi, M. Gionfriddo and R. Rota, "Perfect Octagon Quadrangle Systems-II," Discrete Mathematics, Vol. 312, No. 3, 2012, pp. 614-620. doi:10.1016/j.disc.2011.05.009

[10] S. Kucukcifci and C. C. Lindner, "Perfect Hexagon Triple Systems," Discrete Mathematics, Vol. 279, No. 1-3, 2004, pp. 325-335. doi:10.1016/S0012-365X(03)00278-4

[11] C. C. Lindner and A. Rosa, "Perfect Dexagon Triple Systems," Discrete Mathematics, Vol. 308, No. 2-3, 2008, pp. 214-219. doi:10.1016/j.disc.2006.11.035

[12] L. Gionfriddo, "Hexagon Quadrangle Systems," Discrete Mathematics, Vol. 308, No. 2-3, 2008, pp. 231-241. doi:10.1016/j.disc.2006.11.037

[13] L. Gionfriddo and M. Gionfriddo, "Perfect Dodecagon Quadrangle Systems," Discrete Mathematics, Vol. 310, No. 22, 2010, pp. 3067-3071. doi:10.1016/j.disc.2009.02.029

[14] M. Gionfriddo, L. Milazzo, A. Rosa and V. Voloshin, "Bicolouring Steiner Systems S(2,4,v)," Discrete Mathematics, Vol. 283, No. 1-3, 2004, pp. 249-253. doi:10.1016/j.disc.2003.11.016

[15] S. Milici and G. Ragusa, "Maximum Embedding of an $\mathrm{H}(\mathrm{v}-\mathrm{w}, 3,1)$ into a TS(v, $\lambda)$," Australasian Journal of Combinatorics, Vol. 46, 2010, pp. 121-127.

[16] M. Gionfriddo and C. C. Lindner, "Construction of Steiner Quadruples Systems Having a Prescribed Number of Blocks in Common," Discrete Mathematics, Vol. 34, No. 1, 1981, pp. 31-42. doi:10.1016/0012-365X(81)90020-0

[17] Y. Chang and G. Lo Faro, "Intersection Numbers of Kirkman Triple Systems," Journal of Combinatorial Theory A, Vol. 86, No. 2, 1999, pp. 348-361. doi: $10.1006 /$ jeta. 1998.2948

[18] M. Gionfriddo and Z. Tuza, "On Conjectures of Berge and Chvátal," Discrete Mathematics, Vol. 124, No. 1-3, 1994, pp. 79-86. doi:10.1016/0012-365X(94)90086-8

[19] M. Gionfriddo and S. Milici, "A Result Concerning Two Conjectures of Berge and Chvátal," Discrete Mathematics, Vol. 155, No. 1-3, 1996, pp. 77-79. doi:10.1016/0012-365X(94)00371-O 